\section{Inflammatory Bowel Disease}

\section{Orals}

\section{OTH-12 EFFICACY AND SAFETY OF VEDOLIZUMAB SUBCUTANEOUS FORMULATION FOR ULCERATIVE COLITIS: RESULTS OF THE VISIBLE TRIAL}

\begin{abstract}
${ }^{1}$ WJ Sandborn, ${ }^{2} \mathrm{~F}$ Baert, ${ }^{3} \mathrm{~S}$ Danese, ${ }^{4} \mathrm{Z}$ Krznarić, ${ }^{5} \mathrm{G}$ D'Haens, ${ }^{6} \mathrm{~T}$ Kobayashi, ${ }^{7} \mathrm{X}$ Yao, ${ }^{7} \mathrm{~J}$ Chen, ${ }^{7} \mathrm{~K}$ Kisfalvi, ${ }^{2} \mathrm{~S}$ Vermeire. ${ }^{1}$ University of California San Diego USA; ${ }^{2}$ University Hospitals Leuven, Belgium; ${ }^{3}$ Humanitas University, Milan, Italy; ${ }^{4}$ Clinical Hospital Centre Zagreb, Croatia; ${ }^{5}$ Academic Medical Centre, Amsterdam, Netherlands; ${ }^{6}$ Kitasato University Kitasato Institute Hospital, Tokyo, Japan; ${ }^{7} T a k e d a$ Development Center Americas Inc., Cambridge, USA
\end{abstract}

\subsection{6/gutjnl-2019-BSGAbstracts.119}

Introduction Vedolizumab, a gut-selective, humanised, monoclonal $\alpha 4 b 7$ integrin antibody, is available as an intravenous (IV) formulation to adult patients (pts) with moderately to severely active ulcerative colitis (UC) or Crohn's disease. We present the phase 3 results on a new subcutaneous (SC) formulation for maintenance treatment in UC.

Methods A randomised, double-blind, double-dummy, placebocontrolled, phase 3 trial (NCT02611830) assessed vedolizumab SC as maintenance treatment in adult pts with active UC. An open-label induction with vedolizumab IV $(300 \mathrm{mg})$ was administered at Weeks (Wks) 0 and 2, with disease evaluation at Wk 6. Pts with a clinical response at Wk 6 (complete Mayo score reduction of $\geq 3$ points and $\geq 30 \%$ from baseline [Wk 0] plus reduction in rectal bleeding subscore of $\geq 1$ point or absolute subscore of $\leq 1$ point) were randomised $(2: 1: 1)$ to receive vedolizumab SC (108 $\mathrm{mg}$ every 2 wks), or vedolizumab IV (300 mg every 8 wks) or placebo for up to $52 \mathrm{wks}$. The primary objective was to assess clinical remission (defined as complete Mayo score of $\leq 2$ points and no individual subscore $>1$ point) with vedolizumab SC vs placebo at Wk 52 . Between-group treatment effects were compared using the Cochran-Mantel-Haenszel test with stratification by study randomisation factors (concomitant corticosteroid use, Wk 6 remission status, and prior anti-TNF $\alpha$ failure or immunomodulator use).

Results A total of 383 pts received open-label vedolizumab IV induction. Of those, 216 (56.4\%) experienced clinical response at Wk 6 and entered the maintenance phase. At Wk 52, $46.2 \%$ of pts on vedolizumab SC vs $14.3 \%$ on placebo were in clinical remission $(\mathrm{p}<0.001)$. Similarly, $42.6 \%$ of vedolizumab IV pts were in clinical remission at Wk 52. Subgroup analysis showed clinical remission rates were significantly higher with vedolizumab SC vs placebo in both anti-TNF $\alpha$ naïve pts (vedolizumab $53.7 \%$ vs placebo $18.9 \%, \mathrm{p}<0.001$ ) and anti-TNF $\alpha$-failure pts (vedolizumab $33.3 \%$ vs placebo $5.3 \%, \mathrm{p}=0.023)$. Injection-site reactions were mild $(9.4 \%$ vedolizumab SC vs $0 \%$ placebo pts) none leading to discontinuation. Adverse event (AE) rates, including severe AEs and infections, were similar in the vedolizumab SC and IV groups. The rate of anti-vedolizumab antibodies in the vedolizumab SC group was $5.7 \%$, consistent with $5.6 \%$ for vedolizumab IV. Conclusions Vedolizumab SC $108 \mathrm{mg}$ every 2 wks was efficacious, generally safe and well-tolerated as maintenance therapy in UC pts following induction with vedolizumab IV $300 \mathrm{mg}$ showing an efficacy and safety profile similar to that of the currently available IV formulation.

\section{OTU-22 THIOPURINE WITHDRAWAL DURING REMISSION IN INFLAMMATORY BOWEL DISEASE: RELAPSE AND RECAPTURE RATES IN 72 PATIENTS}

Heather*, Katharine Wade, Parvathy Radhakrishnan, Simon McLaughlin. Royal Bournemouth Hospital, Bournemouth, UK

\subsection{6/gutjnl-2019-BSGAbstracts. 120}

Introduction Long term treatment with thiopurines is associated with an increased risk of opportunistic infection, lymphoma and other malignancies. Treatment withdrawal should be considered in patients who are in deep remission.

To establish the percentage of patients that relapse after withdrawal of azathioprine and time from withdrawal to relapse and recapture rate.

Methods We searched our IBD database for patients where azathioprine (AZA) was withdrawn following a deep remission (defined as faecal calprotectin $(\mathrm{FCP})<200 \mathrm{mg} / \mathrm{kg}$ and/endoscopic remission defined as no ulceration or quiescent inflammation). We reviewed the length of time on AZA, the relapse rates following withdrawal and recapture rate following restarting.

Results 72 patients were identified (36 Crohn's disease (CD); 36 ulcerative colitis (UC). Mean age at stopping $=61.5 \mathrm{yrs}$ (range 21-84). 36 (50\%) male; Median duration of thiopurine use prior to withdrawal $=6.5 \mathrm{yrs}($ range $1.0-14.8) .52(72 \%)$ had FCP measured; mean $=37$ (5-161). Endoscopic remission was confirmed in 36 (50\%). In 3 (18.8\%) FCP was normal with mild inflammation endoscopically.

A total of $28(38.8 \%)$ patients relapsed; 17 UC (60.7\%) $11 \mathrm{CD}$ (39.3\%), mean time until relapse $=16$ months (range 7 days-79 months). Relapse was confirmed endoscopically in $22(78.6 \%)$ and by FCP in 6 (21.4\%). Mean FCP 940 (range 459-2329); 20 (68.9\%) re-started AZA with concomitant prednisolone. Of these $14(70 \%)$ entered clinical remission. 6 patients $(50 \% \mathrm{UC})$ failed to enter remission on re-starting AZA. $3(10.3 \%)$ patients required admission for IV hydrocortisone. A clinical decision was made to start 2 (6.9\%) on methotrexate because of better risk profile in older age. 7 (24.1\%) re-started high dose mesalazine monotherapy. 1 (3.1\%) opted for colectomy (immunosuppresion was not restarted due to a diagnosis of malignant melanoma). And 1 had vedolizumab due to a diagnosis of sarcoma. In total 16 patients $(57.1 \%)$ entered and maintained remission (length of follow up $=$ mean 29.7 months (range 2-120) without requiring surgery or biologics.

Conclusions Our data demonstrate that $38.8 \%$ of patients stopping thiopurine will relapse and $79.4 \%$ of these patients will relapse within 3 years. Reassuringly, $71.5 \%$ achieved remission on re-starting $\mathrm{AZA} /$ methotrexate or mesalazine. Interestingly, 3 of our patients were identified to be in remission with FCP but had mild inflammation on endoscopy. These three patients subsequently relapsed. This raises the possibility of the need for diagnosis of remission endoscopically rather than using FCP alone. This data should aid discussion regarding the safety of withdrawing thiopurine in patients in a long term remission. 